\title{
SÍNDROME DE ZOLLINGER-ELLISON
}

\section{ZOLLINGER-ELLISON SYNDROME}

\author{
Cirilo Luiz de Pardo Mêo Muraro, TCBC-SP ${ }^{1}$ \\ Hercio Azevedo de Vasconcelos Cunha, RCBC-SP ${ }^{2}$ \\ Carlos Eduardo de Freitas Júnior ${ }^{2}$
}

\section{INTRODUÇÃO}

Zollinger e Ellison, em 1955, publicaram trabalho que caracterizava o gastrinoma e apresentava a hipótese de uma pancreatite endócrina originária de hipersecreção gástrica e múltiplas úlceras. Hoje, a enfermidade é definida por hipergastrinemia devida à presença de gastrinoma, levando ao aumento da acidez gástrica, passando a ser chamada de Síndrome de Zollinger-Ellison (SZE) ${ }^{1}$.

O gastrinoma (tumor produtor de gastrina) está localizado na maioria das vezes no pâncreas, podendo existir em outras localizações, como no duodeno ${ }^{2,4}$. A hipersecreção de gastrina produz úlceras pépticas, má digestão, esofagite, duodenojejunite e/ou diarréia ${ }^{1}$.

Em 20\% dos casos a SZE está relacionada com neoplasia endócrina múltipla tipo I (NEM I), que acompanha na maioria das vezes de hiperparatireiodismo $(80 \%)^{1} \mathrm{e}$ em alguns raros casos insulinomas, glucagomas, VIPomas ou outros tumores ${ }^{1,2}$. Além disso, em aproximadamente $20 \%$ dos casos são encontradas metástases a distância e a associação com tumor carcinóide, pela hipergastrinemia, também é descrita ${ }^{1,4}$.

Antes de 1970, a gastrectomia total era o tratamento de escolha para esses pacientes, porém a cura pela ressecção do tumor raramente era relatada ${ }^{4}$. O tratamento hoje é feito com altas doses de bloqueadores da bomba de próton (Omeprazol, Lanzoprazol, Pantoprazol) que conseguem diminuir significativamente a hipersecreção de ácido. $\mathrm{O}$ tratamento cirúrgico é indicado na tentativa de exérese do tumor quando consegue-se a visualização do mesmo pela tomografia computadorizada (TC) ou ressonância magnética (RM), porém o índice de cura é muito baixo, visto que, além dos tumores serem de pequeno tamanho (em média de 0,5 a 1,0cm), muitas vezes são múltiplos ou estão em localização de difícil acesso cirúrgico ${ }^{1,3,4}$. O tratamento cirúrgico é contra-indicado nos pacientes portadores de NEM 1 e/ou metástases a distância ${ }^{1,2,4}$.
Os autores justificam a apresentação do caso pela raridade da síndrome ( 0,1 a 3 casos/um milhão de pessoas $)^{3}$.

\section{RELATO DO CASO}

M.N.S., 49 anos, sexo feminino, solteira, católica, monitora de centro infantil, natural e procedente de Limeira - SP.

Paciente com história de antrectomia com reconstrução a Billroth II por úlceras pépticas com intratabilidade clínica, há quatro anos, mantendo queixas dispépticas. A esofagogastroduodenoscopia (EGD) há três anos evidenciava lesão ulcerada de boca anastomótica com anatomopatológico (AP) revelando borda de lesão ulcerada crônica e ausência de sinais de malignidade. A ultra-sonografia e a tomografia computadorizada de abdome realizadas na mesma época, achavam-se dentro dos limites da normalidade.

Há três anos submeteu-se à degastrectomia com reconstrução a Y de Roux e vagotomia troncular. No intraoperatório evidenciou-se úlcera em anastomase gastrojejunal perfurada e aderida ao peritônio parietal em flanco esquerdo. O AP da lesão revelou úlcera crônica em boca anastomótica e ausência de sinais de malignidade.

Através de acompanhamento ambulatorial, três meses após a cirurgia, apresentava-se emagrecida, descorada $++/ 4+$, com melena ao toque retal. Foi submetida à nova EGD que evidenciou orifício amplo, de aproximadamente $3 \mathrm{~cm}$ de diâmetro, adjacente à anastomose gastroentérica, facilmente transposto pelo aparelho, chegando-se à cavidade com conteúdo semelhante a fezes. Foi realizada, então, a nova cirurgia para correção de fístula gastrojejunocólica, quando foi realizado gastrectomia total com reconstrução a Y de Roux e transversectomia com anastomose término-terminal.

Há dois anos, ultra-sonografia abdominal evidenciou nódulos hepáticos sugerindo metástases, sendo então solicitada tomografia computadorizada de abdome que mostrou

1. Professor Titular da Disciplina e Chefe do Serviço.

2. Residente de Clínica Cirúrgica da PUC-Campinas.

Recebido em 15/6/1999

Aceito para publicação em 22/5/2000

Trabalho realizado na Disciplina de Moléstias do Aparelho Digestivo I (MAD I) e Serviço de Gastroenterologia Cirúrgica do HMCP da Faculdade de Ciências Médicas da Pontifícia Universidade Católica de Campinas - PUC-Campinas. 
seis imagens nodulares hipodensas (metástases), também observadas na ressonância magnética (Figura 1A, 1B, 1C).

Paciente permaneceu um ano e meio sem acompanhamento ambulatorial, retornando há três meses quando foi realizada tomografia computadorizada com microcortes para pâncreas com conclusão de processo expansivo mal delimitado na cauda pancreática e nódulos hepáticos múltiplos, com hipótese diagnóstica de gastrinoma primitivo do pâncreas com metástases hepáticas (Figura 2A, 2B, 2C).

Solicitou-se gastrina sérica, com resultado de $1.532 \mathrm{pg} / \mathrm{ml}$ (normal até $115 \mathrm{pg} / \mathrm{ml}$ ), o que, juntamente com o quadro clínico, história da paciente e os exames de imagens realizados, definiu o diagnóstico da síndrome.

A paciente, seis meses após o diagnóstico, encontrase em bom estado geral, assintomática, com EGD evidenciando gastrectomia total, sem outras alterações, em tratamento medicamentoso com omeprazol 40mg/dia. Não foi optado por tratamento cirúrgico pela irressecabilidade das metástases hepáticas.

\section{DISCUSSÃO}

No presente trabalho os autores apresentam um caso de paciente portadora de úlceras pépticas com intratabilidade clínica, apresentando recidivas mesmo após tratamentos cirúrgicos. O diagnóstico de SZE foi aventado após quatro anos de acompanhamento, com tratamentos clínicos e cirúrgicos, o que também é relatado por Baser I. Hirchowitz, em trabalho publicado no The American Journal of Gastroenterology ${ }^{1,3}$.

Apesar da SZE ser considerada uma enfermidade rara (0,1-3 casos/um milhão de pessoas), é provável que exis- tam mais pacientes com a síndrome, visto que o diagnóstico da mesma nem sempre é lembrado, e que exames endoscópicos e de imagem (TC e RM), na maioria das vezes não mostram alterações, devido ao diminuto tamanho dos tumores e suas variadas localizações ${ }^{3}$.

O diagnóstico da SZE é caracterizado pela presença de úlceras pépticas múltiplas, persistentes, com localizações atípicas, com complicações como sangramento ou perfuração e, em algumas ocasiões (como no presente caso), com fístulas gastroentéricas pós-gastrectomia. Esofagite $(50 \%)$, diarréia, má absorção intestinal e perda de peso também costumam estar presentes. Os exames laboratoriais que justificam a síndrome são a gastrina sérica elevada ( $>115 \mathrm{pg} / \mathrm{ml})$ e a secreção de ácido basal (> 15mEq/h ou maior do que $\mathrm{mEq} / \mathrm{h}$ ).

Antes de considerarmos uma ressecção primária do gastrinoma, o paciente deve ser submetido à ultra-sonografia e TC abdominal. Mesmo sem anatomopatológico comprobatório, a evidência clínica, juntamente com os exames subsidiários, define o diagnóstico da síndrome.

A pesquisa de NEM I sempre deverá ser sempre realizada, com dosagem do cálcio sérico, uma vez que em $20 \%$ dos pacientes com SZE, o gastrinoma está associado à NEM I ${ }^{3}$. Pacientes com NEM I e aqueles com metástases hepáticas ou a distância não são candidatos à cirurgia ${ }^{3}$. Aproximadamente $50 \%$ dos gastrinomas são malignos ${ }^{3,8}$.

Nos pacientes em que a operação é indicada, o que acorre em aproximadamente $10 \%$ dos casos, mesmo com ultra-sonografia, angiografia pancreática e TC intra-operatórios, não se consegue a localização do tumor ${ }^{1}$. Nos casos onde a exérese do tumor é possível, a sobrevida em cinco anos é estimada em menos de $30 \%{ }^{1,5}$. O tratamento
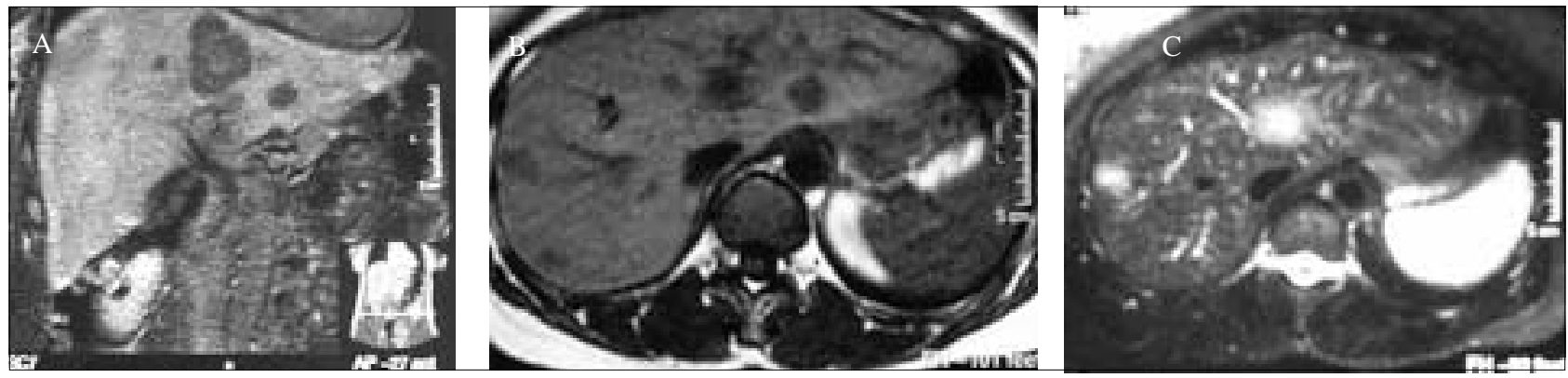

Figura 1 A, B, C - Ressonância Magnética abdominal evidenciando metástases hepáticas.
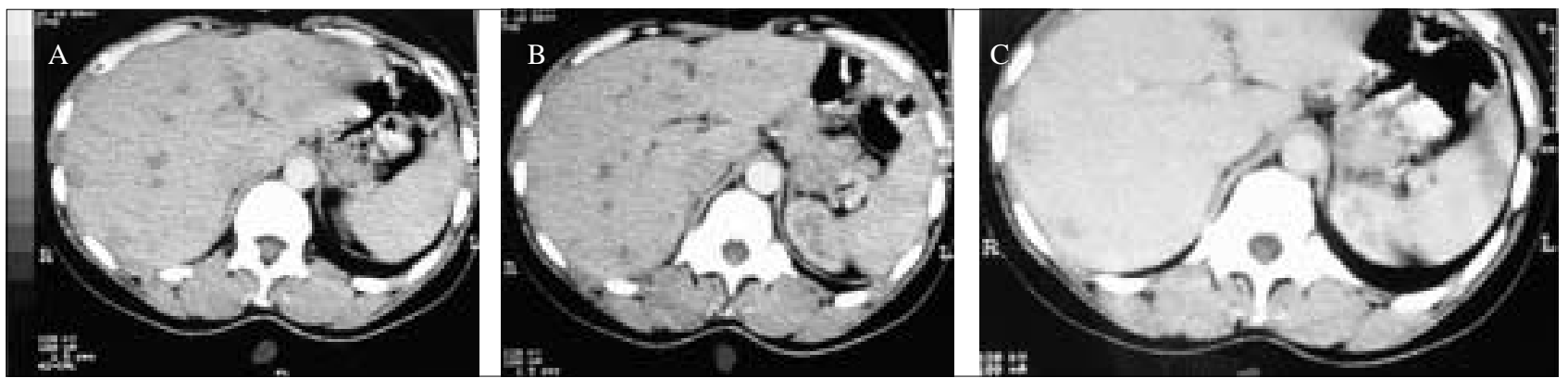

Figura 2A, 2B, 2C - Tomografia computadorizada de pâncreas evidenciando gastrinoma. 
clínico consiste em altas doses de bloqueadores da bomba de próton, como o Omeprazol $(20$ - 160mg/dia) ou Lanzoprazol $(30-165 \mathrm{mg} / \text { dia })^{1}$.

Os autores, a partir deste caso, concluem que, apesar de rara, a SZE deve ser lembrada em pacientes com úlceras pépticas recorrentes e/ou localizações atípicas e nos quais a dosagem de gastrina sérica indica fortemente a presença da enfermidade, podendo direcionar ao diagnóstico da síndrome em menos tempo do que usualmente ocorre.

\begin{abstract}
The authors report a 49 years old, female patient who have been operated on several times (antrectomy with Billroth II reconstruction, partial gastrectomy with troncular vagotomy and total gastrectomy) in the last 5 years for recurrent ulcer disease. Three months ago, an abdomen ultra sound was done showing multiples images that suggested liver metastasis, which was confirmed by CT and RM. Two months ago, one new abdomen CT specifically to pancreas was done showing an expansive process in pancreas. Serial gastrine was $1532 \mathrm{pg} / \mathrm{ml}$ at the time (reference - until 115) and among clinical history and images exams Zollinger-Ellison Syndrome was suggested, a rare disease case.
\end{abstract}

Key Words: Zollinger-Ellison syndrome; Gastrinoma.

\section{REFERÊNCIAS}

1. Hirschowitz BI - Zollinger-Ellison Syndrome: Pathogenesis, Diagnosis, and Management - Am J Gastroenterol, 1997; 92(3): 44-48.

2. Jensen RT - Management of the Zollinger-Ellison Syndrome in patients with Multiple Endocrine Neoplasia Type 1. J Intern Med, 1998; 243(6): 477-488.

3. Jorgensen NR, Rehfeld JF, Bardram L et al. - ProcessingIndependent Analysis in the Diagnosis of Gastrinomas - Scand J Gastroenterol, 1998; 33(4): 379-385.

4. McArthur KE, Richardson CT, Barnett CC et al. - Laparotomy and Proximal Gastric Vagotomy in ZollingerEllison Syndrome: Results of a 16 Year Prospective Study. Am J Gastroenterol, 1996; 99: 1104-1111.
5. Norton JA, Doppman JL, Jensen RT - Curative ressection in patients with Zollinger-Ellison Syndrome: Results of a 10 year Prospective Study. Am Surg, 1992; 215: 703-710.

Endereço para correspondência:

Dr. Cirilo Pardo Mêo Muraro

Av. Andrade Neve, 589

13013-160 - Campinas-SP 